\title{
Recent results on light nuclei production from the NA49 experi- ment
}

\author{
V. I. Kolesnikov ${ }^{1, \star}$ for the NA49 Collaboration \\ ${ }^{1}$ Joint Institute for Nuclear Research, Dubna, Russia
}

\begin{abstract}
Production of $d$ and ${ }^{3} \mathrm{He}$ nuclei in central $\mathrm{Pb}+\mathrm{Pb}$ interactions was studied at five collision energies $\left(\sqrt{s_{N N}}=6.3,7.6,8.8,12.3\right.$, and $\left.17.3 \mathrm{GeV}\right)$ with the NA49 detector at the CERN SPS. Transverse momentum spectra and rapidity distributions were measured. Yields are compared to predictions of statistical models. Phase-space distributions of light nuclei are discussed and compared to those of protons in the context of a coalescence approach. The coalescence parameters $B_{2}$ and $B_{3}$ for $d$ and ${ }^{3} \mathrm{He}$ were determined at all energies.
\end{abstract}

\section{Introduction}

The main goal of the heavy-ion program at the CERN SPS is the study of the properties of hot and dense nuclear matter which can be probed experimentally by measuring particle composition, longitudinal and transverse momentum distributions of different species. The study of light nuclei production is of importance for several reasons. First of all, the mechanism of cluster formation in the interior of the fireball of a heavy-ion collision is not well understood and requires further quantitative investigations. It is likely that a significant fraction of light nuclei near the center-of-mass rapidity (midrapidity) are produced in a late stage of the reaction when the hadronic matter becomes diluted, thus probing the reaction dynamics at the time of the freeze-out. Secondly, in a conventional thermal model, particle multiplicities are predicted dependent on the bulk thermal parameters of the system - the chemical freeze-out temperature $T$, baryochemical potential $\mu_{B}$ and volume $V$. Though recent versions of statistical thermal models describe well hadron abundances in the range of heavy-ion collision energies from about 1 to several $10^{3} \mathrm{GeV}$ per nucleon $[1,2]$, the question was raised whether this approach is justified when applied to the production of nucleon clusters. Because thermal models consider particle yields integrated over the full phase space the lack of results on total multiplicities of light nuclei has up to now prevented a straightforward and quantitative test of the applicability of the statistical model approach to light nuclei production in the SPS energy range.

\section{Data sets and analysis procedure}

The data used in this analysis were collected in years 1996-2002. The NA49 experiment [3] utilized a ${ }^{208} \mathrm{~Pb}$ beam at energies of $20 \mathrm{~A}, 30 \mathrm{~A}, 40 \mathrm{~A}, 80 \mathrm{~A}$ and $158 \mathrm{~A} \mathrm{GeV}$ impinging on a lead target of

^e-mail: Vadim.Kolesnikov@cern.ch 
$224 \mathrm{mg} / \mathrm{cm}^{2}$ thickness corresponding to a $1 \%$ interaction probability. The interaction trigger selected the $12 \%$ most central collisions at $158 \mathrm{~A} \mathrm{GeV}$, at other beam energies the data were recorded with a $7 \%$ central trigger. To ensure a good quality of event and track reconstruction, several cuts were applied to select events and tracks for further analysis. The identification of light nuclei $\left(d,{ }^{3} \mathrm{He}\right)$ was based on momentum, $\mathrm{dE} / \mathrm{dx}$, and time-of-flight measurements. Deuteron candidates were required to have a TOF hit matched to the MTPC track, while the identification of ${ }^{3} \mathrm{He}$ candidates can rely completely on the specific energy loss measurement in the MTPC gas. The raw yields of clusters were corrected for geometrical acceptance, detector efficiency, and for the losses due to the PID selection criteria. The corrections were obtained from Monte Carlo (MC) simulations and from the data itself. More details about the analysis procedure can be found in [4].

\section{Results}

The invariant $p_{t}$ spectra of identified ${ }^{3} \mathrm{He}$ nuclei at five collision energies in different rapidity intervals are shown in figure 1 . The NA49 acceptance for light nuclei is sufficient to examine spectra in several rapidity intervals and their sizes are indicated in figure 1. The experimental distributions are scaled down successively for clarity of presentation and the same scaling factors were used for the rapidity slices located symmetrically with respect to the center of mass rapidity. In order to obtain particle rapidity density ( $\mathrm{dN} / \mathrm{dy})$ we summed the measured points over the $p_{t}$ distributions and added the extrapolations into unmeasured $p_{t}$ regions exploiting information on the spectral shape. The extrapolation amounts to $3-7 \%$ of the $\mathrm{dN} /$ dy value for the ${ }^{3} \mathrm{He}$ spectra near mid-rapidity and increases to almost $40 \%$ for the results at the most forward rapidity. The overall systematic uncertainty for the yields of ${ }^{3} \mathrm{He}$ is of about $6 \%$ at mid-rapidity and approximately $10 \%$ for the most forward rapidity bin. For deuterons the systematic uncertainty varies from $5 \%$ to $15 \%$ depending on rapidity interval.

The cluster yields are plotted in figure 2 as a function of the normalized rapidity $y / y_{\text {beam }}$. All the rapidity distributions are concave and in order to quantify the shape all the data points were fitted with a parabola $a+b\left(y / y_{\text {beam }}\right)^{2}$ (the fits are shown by dashed lines). The ratio of the fit parameters $b / a$ (relative concavity) tends to increase with increasing beam momentum and cluster mass. Such a behavior of the invariant yields versus rapidity was earlier observed at AGS energies [5], where the relative concavity of the yield of clusters with atomic mass number from $A=2$ to 4 progressively increases with $A$.

The total yield was then obtained by summing the measured values with the integral of the corresponding extrapolation function over the unmeasured region. To extrapolate the integral of $\mathrm{dN} / \mathrm{dy}$ to full phase space, averaging of two different parameterizations of the rapidity spectra were employed as explained in [6]. The extrapolation accounts from $30 \%$ to $63 \%$ and from $20 \%$ to $85 \%$ of the total $(4 \pi)$ yield for ${ }^{3} \mathrm{He}$ and $d$, respectively, depending on the collision energy. The estimates for the $4 \pi$ yield of deuterium and helium nuclei are plotted in figure 3 with red squares.

In the framework of a statistical thermal model the abundance $N_{C}$ of a nucleon cluster of mass $m$, degeneracy factor $g$, charge $q$, and baryon number $B$ is given by

$$
N_{C}=\frac{g V}{\pi^{2}} m^{2} T K_{2}(m / T) \exp \left(\frac{B \mu_{B}+q \mu_{q}}{T}\right),
$$

where $V, T, \mu_{B}, \mu_{q}$, and $K_{2}$ are the source volume, temperature, baryochemical potential, charge potential, and Bessel function of the second kind. Such models have been able to reproduce the multiplicities of different types of particles in elementary and heavy-ion interactions. There are several parameterizations for the thermal fireball parameters $T, \mu_{B}$, and $V$ (or equivalently the fireball radius $R$ ) over a wide range of nuclear collision energies from AGS to LHC [7-10]. Using averages of these 


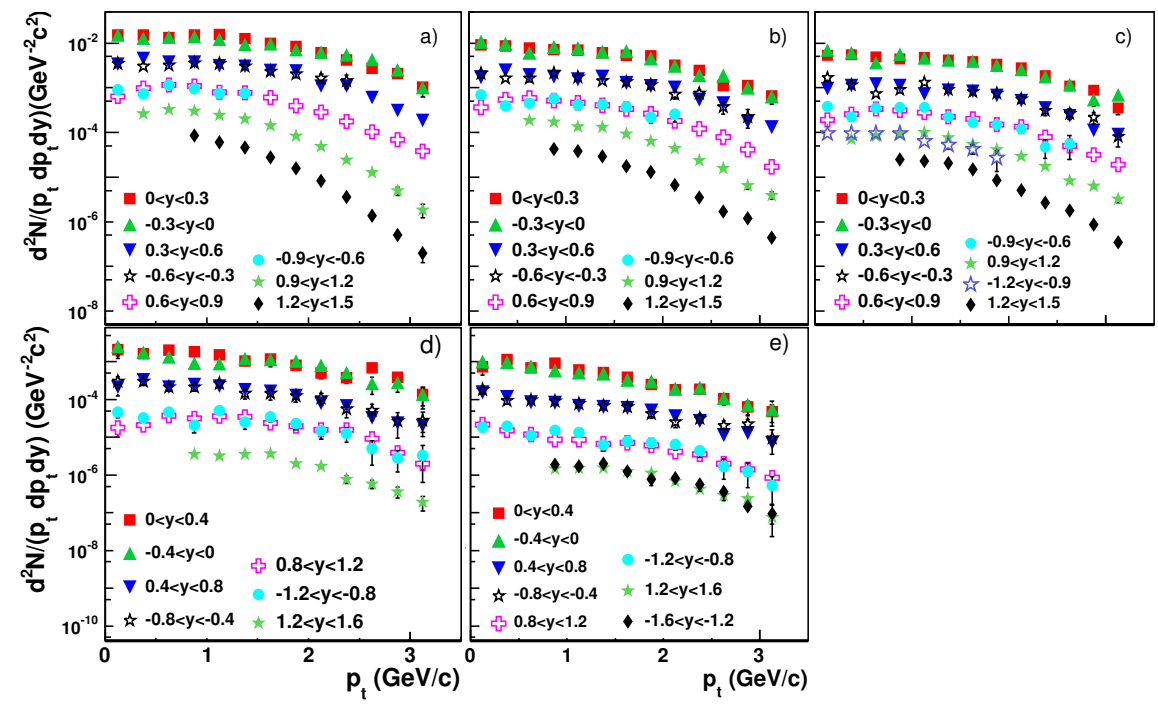

Figure 1. Invariant $p_{t}$ spectra of ${ }^{3} \mathrm{He}$ at $20 A$ (a), $30 A$ (b), $40 A$ (c), $80 A$ (d), and $158 A \mathrm{GeV}$ (e). Only statistical errors are shown. The distributions near mid-rapidity are drawn to scale, other spectra are scaled down by successive powers of 5 for clarity. The same scaling factor is used for two rapidity slices that are symmetric about mid-rapidity $(y=0)$.
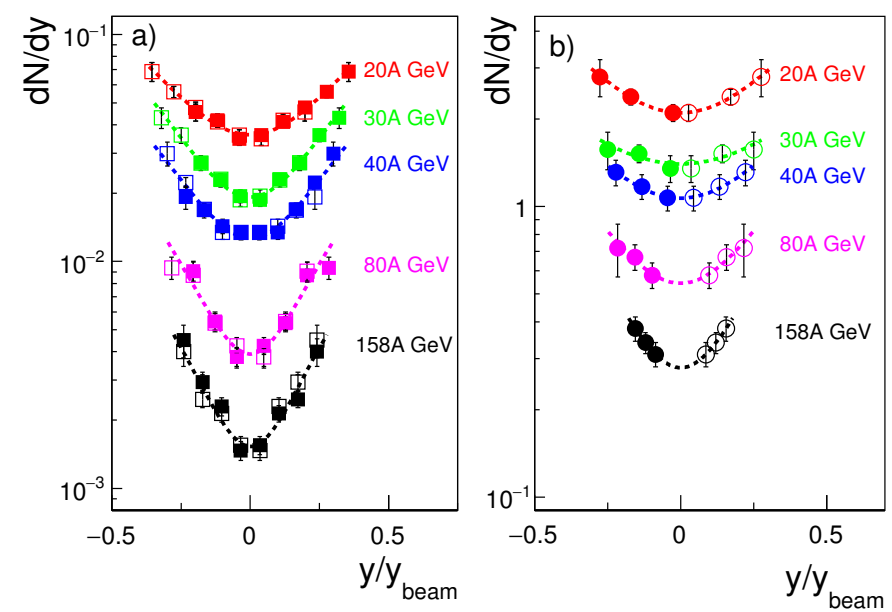

Figure 2. Rapidity distributions for ${ }^{3} \mathrm{He}$ (a) and deuterons (b) from central $\mathrm{Pb}+\mathrm{Pb}$ collisions at $20 A-158 A \mathrm{GeV}$ as a function of normalized rapidity $y / y_{\text {beam }}$. The solid symbols show the measurements and the open ones are the data points reflected around mid-rapidity. Dashed lines indicate parabolic fits to the rapidity spectra.

fireball parameters the mean multiplicities of $d$ and ${ }^{3} \mathrm{He}$ were computed at all five collision energies according to 1 . The results are plotted in figure 3 with blue circles. As it can be seen, thermal model calculations are capable of reproducing the energy dependence of the cluster multiplicities not only qualitatively but also quantitatively. The deviation of the calculations from the measured abundances does not exceed 2 standard deviations (see insets in figure 3). It appears at first glance that such a good agreement between experimental data on cluster production and thermodynamical model calculations 

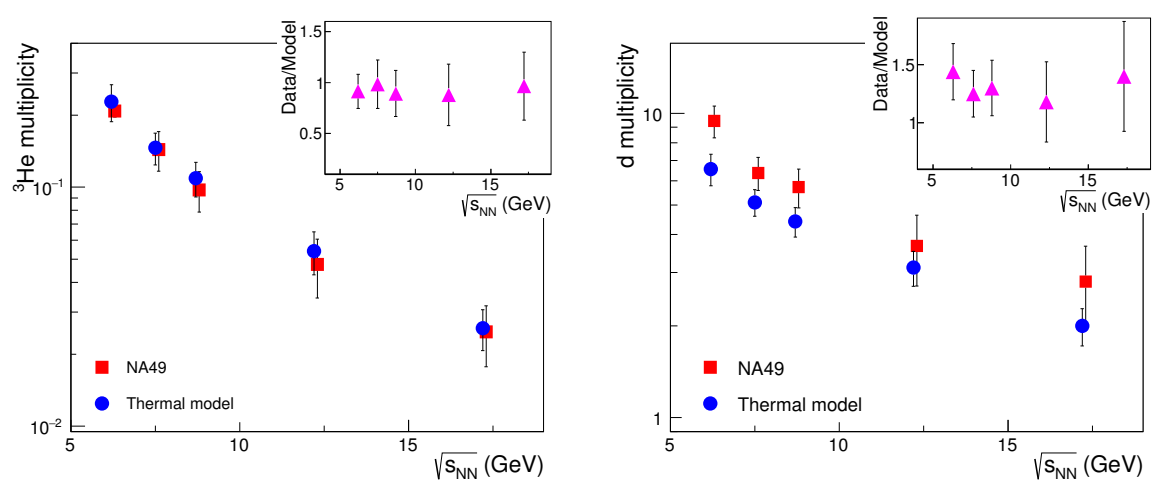

Figure 3. Total yield of ${ }^{3} \mathrm{He}$ (left panel) and deuterons (right panel) in central $\mathrm{Pb}+\mathrm{Pb}$ collisions at $20 \mathrm{~A}-158 \mathrm{~A} \mathrm{GeV}$. The NA49 data are shown by red squares, thermal model calculations are indicated by blue circles. The insets show the ratio of the experimental data to the thermal model predictions.

looks surprising since one expects that chemical equilibrium of compound nucleon systems with the environment reaches later in time than the chemical freeze-out in the fireball has happened. The more diluted and colder matter is needed to make a net (fusion minus decomposition) rate positive for bound nucleon systems of several MeV binding energy. It has been recognized, however, that the light nuclei production process is basically determined by the entropy per baryon of the medium that is fixed at the chemical freeze-out $[11,12]$. During further isentropic expansion stage a dynamic equilibrium has been reached for the light nuclei population: the loss due to disintegration are balanced by the gain in newly produced clusters.

In figure 4 (left panel) is plotted the average transverse energy $\left\langle m_{t}\right\rangle-m$ for ${ }^{3} \mathrm{He}$ as a function of normalized rapidity $y / y_{\text {beam }}$ at five collision energies. As one can see, all the rapidity spectra follow a bell-like shape and we used Gaussian fits to get a mid-rapidity estimate for $\left\langle m_{t}\right\rangle-m$. In the right panel of figure 4 we present our results for the mid-rapidity $\left\langle m_{t}\right\rangle-m$ for protons and light nuclei from central $\mathrm{Pb}+\mathrm{Pb}$ collisions at $20 A-158 A \mathrm{GeV}$. The data points for protons were taken from $[13,14]$, the values for $d$ and ${ }^{3} \mathrm{He}$ were obtained in this study. Evidently, $\left\langle m_{t}\right\rangle-m$ rises approximately linearly with mass at all collision energies. These results may look surprising because it seems unlikely that objects of a few $\mathrm{MeV}$ binding energy per nucleon are participating in multiple thermalization collisions which generate the common velocity field inside fireballs of about 120-140 MeV temperature. On other hand, it was demonstrated in [15] that in the framework of the coalescence approach the choice of a suitable parameterizations for the spatial dependence of the single nucleon density can reproduce the observed mass dependence of the $\left\langle m_{t}\right\rangle-m$ of composites. For example, an interplay between a linear collective flow profile and a uniform density distribution gives an effective temperature rising linearly with mass.

In a coalescence approach $[16,17]$ the invariant yield $N_{A}$ of clusters with charge $Z$ and atomic mass number $A$ is related to the product of the yields of protons $N_{p r}$ and neutrons $N_{n}$ through the coefficient $B_{A}$, the so-called coalescence parameter:

$$
E_{A} \frac{d^{3} N_{A}}{d^{3} P_{A}}=B_{A}\left(E_{p r} \frac{d^{3} N_{p r}}{d^{3} p}\right)^{Z}\left(E_{n} \frac{d^{3} N_{n}}{d^{3} p}\right)^{A-Z},
$$

where $p=P_{A} / A$. Assuming that the ratio of neutrons to protons is unity, $B_{A}$ is then calculated by dividing the cluster yield at a given momentum $P_{A}$ by the A-th power of the proton yields at 

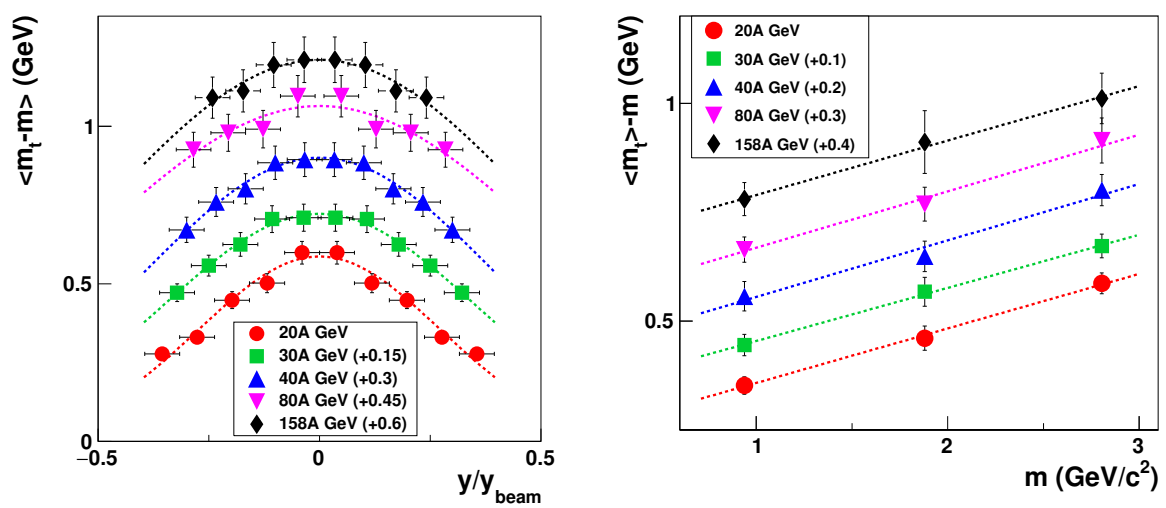

Figure 4. Left: mean transverse mass for ${ }^{3} \mathrm{He}$ as a function of $y / y_{\text {beam }}$ from central $\mathrm{Pb}+\mathrm{Pb}$ at $20 A-158 \mathrm{~A} \mathrm{GeV}$. Dashed lines indicate fits to a Gaussian. Right: mass dependence of $\left\langle m_{t}\right\rangle-m$ in central $\mathrm{Pb}+\mathrm{Pb}$ collisions at 20A-158A GeV. Linear fits to the data points are indicated by dashed lines.

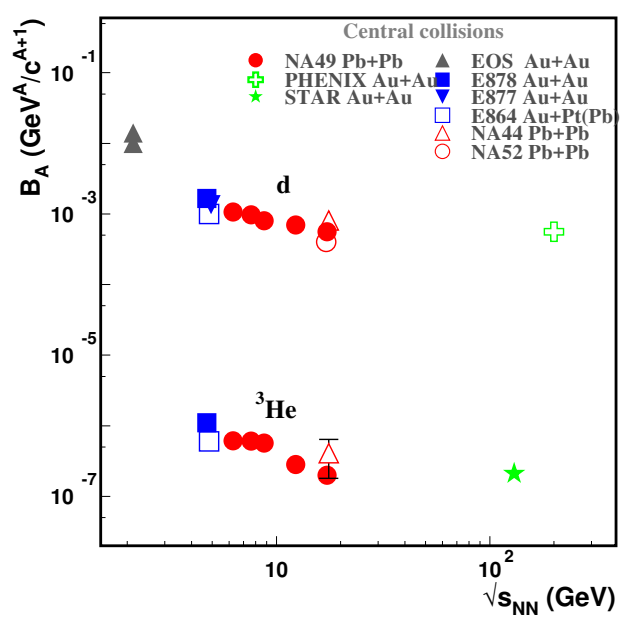

Figure 5. Coalescence parameters $B_{2}$ and $B_{3}$ from central A + A collisions.

$P_{A} / A$. Figure 5 compares the results for $B_{2}$ and $B_{3}$ at $p_{t}=0$ from our study (red filled circles in figure 5) to experimental data from the Bevalac [18], AGS [5, 19], SPS [20, 21], and RHIC [2224]. One concludes from this compilation that the coalescence parameters decrease only slowly with $\sqrt{s_{N N}}$ over a broad range of collision energies: within the SPS energy range the variation of the coalescence parameter is less than $40 \%$ and $60 \%$ for $B_{2}$ and $B_{3}$, respectively. In the framework of thermal models of cluster production $[25,26]$ the coalescence parameter is a measure of the source size: $B_{A} \approx(1 / V)^{A-1}$. Thus, the observed energy dependence of $B_{A}$ implies that the transverse size of the emitting source does not change much in this energy domain. This behavior is consistent with that found in two-pion interferometry measurements [27]. 


\section{Summary}

We present results on the production of $d$ and ${ }^{3} \mathrm{He}$ nuclei in central $\mathrm{Pb}+\mathrm{Pb}$ collisions at $20 A$ $158 A \mathrm{GeV}$ recorded with the NA49 detector at the CERN SPS. The spectra for ${ }^{3} \mathrm{He}$ cover a wide range of rapidity and transverse momentum. Cluster yields were determined and exhibit a concave shape as function of rapidity with an increase of the degree of concavity for heavier systems. The yields of $d$ and ${ }^{3} \mathrm{He}$ integrated over the full phase space agree with thermal model predictions at all collision energies. The transverse mass spectra of clusters were measured and the average values $<m_{t}>-m$ were found to increase linearly with the mass: this behavior favors a combination of a box density profile with a linear velocity profile in the source of the clusters. The coalescence parameters $B_{2,3}$ were derived showing a weak energy dependence. This observation suggests only a small increase of the freeze-out volume from AGS to RHIC energies.

\section{References}

[1] F. Becattini, J. Cleymans, A. Keränen, E. Suhonen, and K. Redlich, Phys. Rev. C 64, 024901 (2001)

[2] A. Andronic, P. Braun-Munzinger, and J. Stachel, Nucl. Phys. A 772, 167 (2006)

[3] S. V. Afanasiev et al. (NA49 Collaboration), Nucl. Instrum. Meth. A430, 210 (1999)

[4] T. Anticic et al. (NA49 Collaboration) Phys. Rev. C 94, 044906 (2016)

[5] T. A. Armstrong et al. (E864 Collaboration), Phys. Rev. C 61, 064908 (2000)

[6] T. Anticic et al. (NA49 Collaboration), Phys. Rev. C 85, 044913 (2012)

[7] A. Andronic, P. Braun-Munzinger, and J. Stachel, Phys. Lett. B 673, 142 (2009)

[8] J. Cleymans, H. Oeschler, K. Redlich, and S. Wheaton, Phys. Rev. C 73, 034905 (2006)

[9] F. Becattini, M. Gaździcki, A. Keränen, J. Manninen, and R. Stock, Phys. Rev. C 69, 024905 (2004)

[10] V. Vovchenko, V. V. Begun, and M. I. Gorenstein, arXiv:nucl-th/1512.08025v1

[11] P. J. Siemens and J. I. Kapusta, Phys. Rev. Lett. 43, 1486 (1979)

[12] D. Hahn and H. Stöcker, Nucl. Phys. A 476, 718 (1988)

[13] C. Alt et al. (NA49 Collaboration), Phys. Rev. C 73, 044910 (2006)

[14] T. Anticic et al. (NA49 Collaboration), Phys. Rev. C 83, 014901 (2011)

[15] A. Polleri, J. P. Bondorf, and I. N. Mishustin, Phys. Lett. B 419, 19 (1998)

[16] S. T. Butler and C. A. Pearson, Phys. Rev. 129, 836 (1963)

[17] A. Schwarzschild and C. Zupancic, Phys. Rev. 129, 854 (1963)

[18] L. Ahle et al. (E802 Collaboration), Phys. Rev. C 60, 064901 (1999)

[19] J. Barrette et al. (E877 Collaboration), Phys. Rev. C 61, 044906 (2000)

[20] G. Ambrosini et al. (NA52 Collaboration), Phys. Lett. B 417, 202 (1998)

[21] I. G. Bearden et al. (NA44 Collaboration), Eur. Phys. J. C 23, 237 (2002)

[22] C. Adler et al. (STAR Collaboration) Phys. Rev. Lett. 87, 262301 (2001)

[23] S. S. Adler et al. (PHENIX Collaboration) Phys. Rev. Lett. 94, 122302 (2005)

[24] I. Arsene et al. Phys. Rev. C 83, 044906 (2011)

[25] J. I. Kapusta, Phys. Rev. C 21, 1301 (1980)

[26] A. Z. Mekjian, Phys. Rev. C 17, 1051 (1978)

[27] C. Alt et al. (NA49 Collaboration), Phys. Rev. C 77, 064908 (2008) 